\title{
4 Methoden
}

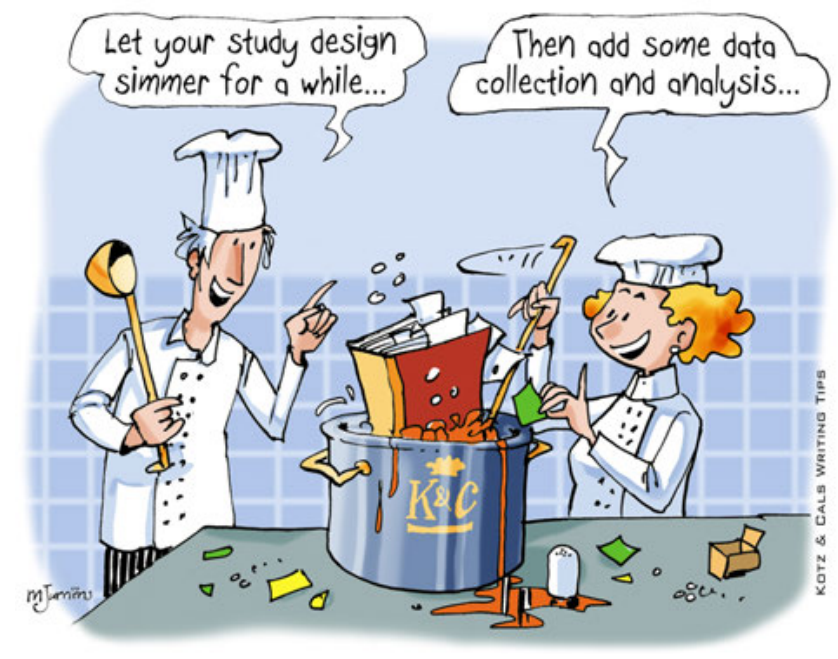

Tipp 4 - Methoden: erstelle ein Kochrezept mit den Zutaten der Studie!

\section{Was Sie wissen sollten}

Würden Sie eine Forschungsstudie wie ein Gourmetgericht des Wissens betrachten, wäre der Methodenabschnitt eines Artikels wie ein Rezept, das alle notwendigen Zutaten der Studie auflistet und angibt, wie diese während des Kochens zubereitet werden müssen. Idealerweise ermöglicht es, das Gericht mit dem gleichen Ergebnis nochmals zuzubereiten. Der Abschnitt Methoden verbindet die Abschnitte Einleitung und Ergebnisse eines Artikels, um einen klaren inhaltlichen Aufbau zu schaffen. Er sollte den naheliegenden Ansatz zur Beantwortung der Fragestellung präsentieren und die Struktur definieren, in der die Ergebnisse später aufgezeigt werden.

Der Methodenabschnitt des Artikels einer originären, quantitativen Studie besteht üblicherweise aus vier Grundelementen: Studiendesign, Studienrahmen und -teilnehmer, Datensammlung und Datenanalyse. Solche Untertitel werden häufig zur Strukturierung des Abschnitts verwendet (die jeweilige Zeitschrift kann spezifische Anleitungen zu Untertiteln geben). Im Falle von Forschung an Menschen muss die zuständige Ethikkommission ebenfalls genannt werden, ge- 
nauso wie eine eventuelle Registrierung der Studie; beides mit entsprechenden Identifikationsnummern.

\section{Was Sie tun sollten}

Beginnen Sie mit der Entwicklung einer Gliederung anhand der Grundelemente des Methodenabschnitts (siehe Teil 1: Anfangen zu Schreiben). Beziehen Sie sich - falls verfügbar - auf ein veröffentlichtes Protokoll oder einen zuvor publizierten Artikel des gleichen Forschungsprojekts für zusätzliche Informationen zu den Methoden. Dies ermöglicht Ihnen, den Methodenabschnitt knapp und präzise zu gestalten. Sorgen Sie jedoch dafür, dass alle wesentlichen Informationen enthalten sind, die der Leser zum Verständnis benötigt, wie die Hauptergebnisse in diesem Artikel abgeleitet wurden.

Erwähnen Sie das Design der vorliegenden Studie, wie randomisierte kontrollierte Studie, prospektive/retrospektive Kohortenstudie, Fall-Kontroll-Studie oder Querschnittsbefragung. Falls Sie es schwierig finden, Ihre Studie einem bestimmten Designtyp zuzuordnen, versuchen Sie, die Kernbestandteile des Designs zu beschreiben, beispielsweise ob es eine interventionelle oder observationelle Studie war und ob Daten im Längsschnitt oder Querschnitt erhoben wurden.

Erklären Sie, wann und wo die Studie durchgeführt wurde, wie die Stichprobe gezogen oder ausgewählt wurde und welche Einschluss-/Ausschlusskriterien angewandt wurden. Geben Sie eine Berechnung der Stichprobengröße für Studien an, die zur statistischen Prüfung einer spezifischen Hypothese eingerichtet wurden.

Definieren Sie hinsichtlich der Datensammlung genau, welche Exposition (z. B. kritische Lebensereignisse) oder Intervention (z. B. kognitive Verhaltenstherapie) Sie untersucht haben, welche Ergebnisse Sie gemessen haben (z. B. Depression), wie Sie diese gemessen haben (z. B. unter Verwendung einer selbstberichteten Depressionsskala) und wann die Messungen durchgeführt wurden (z. B. während des Screenings und der Nachbeobachtung nach 12 Monaten). Zitieren Sie ursprüngliche Forschungsarbeiten zu bestehenden Messinstrumenten, die Sie verwendet haben, und geben Sie an, falls Sie speziell für Ihre Studie ein Messinstrument entwickelt haben. Geben Sie Details zu Messeigenschaften (Reproduzierbarkeit, Validität und Veränderungssensitivität), wenn diese von entscheidender Bedeutung für die Interpretation der Hauptergebnisse sind. Wenn Sie verschiedene Messungen verwendet haben, besteht eine nützliche Reihenfolge darin, mit dem Studienendpunkt (oder abhängigen Variable) 
zu beginnen, gefolgt von den Expositionsmessungen (oder wichtigsten, unabhängigen Variablen) und möglichen Kovariaten und Confoundern.

Stimmen Sie den Absatz zur Datenanalyse mit der Fragestellung ab. Wenn Sie in Ihrer Einleitung eine primäre Fragestellung sowie eine oder mehrere, zusätzliche sekundäre Fragestellungen präsentieren, beginnen Sie den Methodenabschnitt mit den Erläuterungen zur Primäranalyse, gefolgt von den Sekundäranalysen. Beschreiben Sie statistische Techniken in ausreichendem Detail, denn Sie können nicht davon ausgehen, dass Leser anhand des Namens einer Technik verstehen, was Sie genau gemacht haben. Legen Sie die Definition und Operationalisierung der abhängigen und wichtigsten unabhängigen Variablen, die Verwendung von Kovariaten und Confounders (d.h. ob und wie Sie Ihre Analyse adjustiert haben) und die Handhabung fehlender Daten deutlich dar. Seien Sie ehrlich und klar welche Analysen zur Überprüfung Ihrer Hypothese Sie von vornherein definiert haben, und welche zusätzlichen Analysen Sie explorativ durchgeführt haben (post hoc). Erwägen Sie, weitere Details zu den Methoden in einem reinen online Anhang des Artikels oder auf einem web-basierten Verwahrungsort $\mathrm{zu}$ präsentieren (wie dem Open Science Framework). Vermeiden Sie, im Methodenabschnitt Ergebnisse zu präsentieren, wie die Anzahl von Teilnehmern die rekrutiert und nachbeobachtet wurden.

Da es verschiedene Arten geben kann, eine Forschungsfrage zu beantworten, versuchen Sie bei Bedarf zu erklären, warum Sie bestimmte methodische Entscheidungen getroffen haben und warum Sie der Meinung sind, dass diese im gegebenen Kontext die beste Option waren. Sie können die Glaubhaftigkeit Ihrer Methoden beweisen, indem Sie frühere Forschungsstudien zitieren.

Nachdem Sie den Abschnitt zu den Methoden konzipiert haben, sollten Sie sich fragen: „Wäre ein anderer Forscher in der Lage, meine Studie mit den von mir in diesem Artikel bereitgestellten Informationen zu replizieren?“ Überprüfen Sie außerdem, ob der Abschnitt redundante Informationen enthält, die zum Verständnis des Erzählstrangs nicht notwendig sind. Diese Prüfung ist besonders wichtig, wenn der Artikel einer von vielen ist, die aus einem größeren Forschungsprojekt stammen. Beschreiben Sie nur Methoden, deren Ergebnisse Sie später präsentieren. 


\section{Checkliste für die Methoden}

- Nehmen Sie grundlegende Informationen zu Studiendesign, Studienrahmen und -teilnehmer, Datensammlung, Datenanalyse und der zuständigen Ethikkommission auf

- Beziehen Sie sich auf frühere Veröffentlichungen des gleichen, übergreifenden Forschungsprojektes - wie einem Studienprotokoll - für zusätzliche Informationen (falls zutreffend)

- Erwägen Sie die Bereitstellung detaillierterer Informationen zu den Methoden als ergänzende Unterlagen, die im Internet verfügbar sind

- Fragen Sie sich: „Wäre ein anderer Forscher in der Lage, meine Studie mit den von mir in diesem Artikel bereitgestellten Informationen zu replizieren?“ 